\title{
Modifying RANKL/OPG mRNA Expression in Differentiating and Growing Human Primary Osteoblasts
}

Authors

Affiliations
M. Giner ${ }^{1}$, M. J. Montoya ${ }^{2}$, M. A. Vázquez ${ }^{2}$, M. J. Rios², R. Moruno ${ }^{2}$, M. J. Miranda , R. Pérez-Cano ${ }^{1,2}$

${ }^{1}$ Bone Metabolism Unit, Internal Medicine, University Hospital “Virgen Macarena” Seville, Spain

${ }^{2}$ Medicine Department, University of Seville, Spain
Key words

- human osteoblast culture

- 17- $\beta$-estradiol

- 1,25dihydroxyvitamin D3

- osteoporosis

- osteoarthritis received 29.10.2007

accepted 19.05.2008

\section{Bibliography}

DOI 10.1055/s-0028-1082083

Published online:

October 17, 2008

Horm Metab Res 2008;

40: 869-874

(c) Georg Thieme Verlag KG

Stuttgart - New York

ISSN 0018-5043

\section{Correspondence}

\section{Giner}

Bone Metabolism Unit Internal Medicine

University Hospital

Av. Dr. Fedriani s/n

41018 Seville

Spain

Tel.: + 34/954/55 661617

Fax: $+34 / 954 / 556617$

merce_giner@yahoo.es

\section{Abstract}

$\nabla$

The OPG/RANKL system in primary cultures of human osteoblasts has been studied by different authors. However, very few studies have been performed on gene expression of RANKL and OPG at different stages of maturation on human osteoblast cultures. The effect of $17-\beta$-estradiol and 1,25dihydroxyvitamin D3 on the OPG/RANKL system is not known during the different states of cellular maturation. In this work we quantified OPG and RANKL protein levels (ELISA) and the mRNA of OPG, RANKL, collagen type I, alkaline phosphatase, and osteocalcin (semi-quantitative RT-PCR) in human osteoblasts. We analyzed these in basal conditions and after incubation

\section{Introduction}

$\nabla$

Bone turnover is a continuous physiological process depending on the balance between bone formation and resorption. This balance involves the coordinated regulation and interaction of two cell types: the osteoclast $(\mathrm{OC})$ and the osteoblast (OB) $[1,2]$. The OPG/RANKL/RANK system is fundamental for regulation between the OBs and OCs $[3,4]$

Previous in vitro studies of OPG/RANK/RANKL regulation have used stromal cells, immature primary osteoblasts, and osteoblast cell lines. Recent studies using cell lines induced to differentiate into a more mature osteoblastic phenotype have indicated that RANKL and OPG expression levels can be altered $[5,6]$. However, expression models of RANKL and OPG have not been established during primary human osteoblastic differentiation. Only Thomas et al. [7] have demonstrated that RANKL and OPG expression is different during the growth of murine osteoblastic cells.

An OB cell has different levels or stages of maturity during its growth. At each stage the pheno- with 17- $\beta$-estradiol and 1,25dihydroxyvitamin D3 in the first and second phases. We found that OPG secretion and expression levels increased throughout cellular growth. RANKL proteins were detected only in the first stage, and the expression increased throughout the first phase. Thus, the RANKL/OPG ratio was higher in immature osteoblasts than in mature osteoblasts. The evolution of RANKL gene expression was related to collagen I and alkaline phosphatase, while OPG was related to osteocalcin. We observed no modifications after estradiol and 1,25dihydroxyvitamin D3 treatment. Our results suggest that the $\mathrm{OB}$ is a positive stimulator at precocious stages of differentiation on osteoclastogenic modulates.

type, including the morphologic appearance and biosynthetic activity, is different. OB differentiation includes three distinct periods: 1) Growth (proliferation) and extracellular matrix (ECM) biosynthesis, 2) ECM development and maturation, and 3) ECM mineralization. During the period of active proliferation, many genes are expressed, such as cell cycle genes (c-fos, c-myc, histone) and those of extracellular matrix proteins (procollagen I, fibronectin). This is followed by a stage of matrix maturation characterized by a high expression of bone alkaline phosphatase. When mineralization begins, genes for proteins such as osteocalcin, bone sialoprotein and osteopontin are expressed $[8,9]$.

The effects of 1,25dihydroxyvitamin D3 on OB have been well characterized in rats and human osteoblast cultures; in both cases, stimulation and inhibition have been described in genes related to the differentiation of osteoblastic phenotypes $[10,11]$. It is well established that estrogens influence osteoblastic growth and differentiation $[12,13]$. Nevertheless, the simultaneous action of both stimuli has not been described. 
Therefore the main aim of this study was to examine OPG and RANKL expression and secretion in primary cultures of human OBs throughout their developmental sequences. The second point was to analyze the response of the RANKL/OPG system to 17 - $\beta$-estradiol and 1,25dihydroxyvitamin D3 throughout the different maturation stages. The final goal was to relate the OPG and RANKL gene expression with the expression of other genes implicated in bone structural proteins such as osteocalcin (BGP), collagen type 1 (COL 1 ), and alkaline phosphatase (ALP) during the proliferation and maturation of osteoblasts.

\section{Materials and Methods \\ $\nabla$ \\ Cell culture}

Primary human $\mathrm{OB}$ (hOB) cells were isolated from bone fragments taken from four patients ( 3 women and 1 man, aged 58-72 years), without bone metabolic disease, who had undergone knee or hip arthroplasty due to the diagnosis of osteoarthritis. Ethical approval had been obtained from the local Research Ethics Committee. Briefly, trabecular bone was cut into pieces (1-2 mm) and thoroughly rinsed with PBS six times. The cells from each donor were examined individually, they were incubated in a humidified $\mathrm{CO}_{2}$ incubator at $37^{\circ} \mathrm{C}$, and the medium was changed twice a week until confluence was achieved. This was defined as first passage cells. hOB was cultured in $\alpha$-DMEM supplemented with $10 \%$ FCS and antibiotics. In all the cultures, $>85 \%$ of the cells showed intense staining for alkaline phosphatase activity. Trypan blue was used to screen out dead cells after harvesting. We tested cells at 14 and $28 \mathrm{~d}$ and at confluence, and then 300000 cells/plate were sub-cultured and tested again after 14 and $28 \mathrm{~d}$ in the second passage. We confirmed the osteoblastic phenotype by using alkaline phosphatase staining.

\section{Histochemical detection of ALP}

After rinsing monolayer cells with PBS, the cells were fixed in $3.7 \%$. formaldehyde and $90 \%$ ethanol solution for $2 \mathrm{~min}$ and washed in TBS for $10 \mathrm{~min}$. Then, the cells were stained with fast 5-bromo-4-chloro-3-indolyl phosphate and nitroblue tetrazolium (BCIP/NBT) alkaline phosphatase substrate (Amresco, Ohio, USA) for $10 \mathrm{~min}$ at room temperature. The reaction was stopped by removing the substrate solution and washing with distilled water.

\section{Cell treatment with $\mathrm{E} 2$ and vitD}

Confluent hOB cultures were then washed and incubated in a serum-free medium for $24 \mathrm{~h}$. The concentration used for each agent was as follows: $10^{-6} \mathrm{M} 17-\beta$-estradiol and $10^{-8} \mathrm{M} 1$,25dihydroxyvitamin D3.

\section{Measurement of OPG secretion}

OPG levels were analyzed using an ELISA (Immundiagnostik, Bensheim and Biomedia, Vienna). Briefly, the samples and biotinylated antibody against OPG reacted simultaneously with the pre-coated antibody on the microtiter plate; in a second step, streptavidin-peroxidase, which reacts with the detection antibody, was added, and the solid phase was incubated with the substrate, TMB. An acidic stopping solution was subsequently added. A dose-response curve of the absorbance units (at $450 \mathrm{~nm}$ ) versus concentration was generated. The OPG present in samples was determined directly from the calibration curve.
The detection limit was $0.14 \mathrm{pmol} / 1$, the intra-assay variation was $4 \%$, and the inter-assay variation was $<10 \%$. Protein concentrations were normalized to the number of cells.

\section{Measurement of sRANKL secretion}

sRANKL levels were analyzed using an ELISA (Immundiagnostik, Bensheim and Biomedica, Vienna). In a first step, the sample and biotinylated anti-sRANKL detection antibodies were pipetted into the wells. Human SRANKL, if present in the sample, binds to the pre-coated recombinant osteoprotegerin (OPG) and forms a sandwich within the detection antibody. Streptavidin-HRP conjugate was added to the wells, and then TMB was added as a substrate. A dose-response curve of absorbance units (at $450 \mathrm{~nm}$ against $690 \mathrm{~nm}$ or $620 \mathrm{~nm}$ as a reference) versus concentration was generated. The sRANKL present in the samples was determined directly from the calibration curve. The detection limit was $0.08 \mathrm{pmol} / 1$, the intra-assay variation was $4 \%$, and the interassay variation was $9 \%$. Protein concentrations were normalized to the number of cells.

\section{Semi-quantitative RT-PCR for the mRNAs of OPG, RANKL, ALP, COLI, and BGP}

The cells were lysed and the RNA was isolated using a High Pure RNA Isolation Kit (Roche, Germany) according to the manufacturer's instructions. Total RNA was quantified by measuring the OD at $260 \mathrm{~nm}$. An amount of $0.2 \mu \mathrm{g}$ was amplified by RT-PCR using a Titan One Tube Kit (Roche, Germany); primers and conditions are presented in Table 1. Amplified products were analyzed by $2 \%$ agarose gel electrophoresis and visualized with ethidium bromide. Band densities were measured using a Scion Image 4.0.2. (Maryland, USA) computer software program. The relative expression of the different transcripts was calculated as a ratio to the $\beta$-actin signal.

\section{Statistical analysis}

Statistical comparisons were carried out by ANOVA using the SPSS 14.0. software (Illinois, USA). The correlations between variables were carried out by Pearson coefficient. The critical value for significance was $\mathrm{p}<0.05$.

\section{Results}

$\nabla$

Primary human osteoblast cultures progressed throughout the osteoblastic development sequence. All the cultures behaved homogenously, and cellular confluence was reached between 33 and $35 \mathrm{~d}$ after starting the culture.

\section{Osteoclastogenesis regulatory proteins}

OPG protein secretion was detected at all the time points analyzed, although the higher levels were reached at $14 \mathrm{~d}$ of the second passage, with a significant increase regarding the first study point $(p=0.003)$. ( $\bullet$ Fig. 1A).

sRANKL protein secretion was detected only in the initial phase of osteoblastic proliferation, with a decrease of $96 \%$ at day 28 of the first passage with respect to the initial value, while it was not detectable in the rest of the determinations ( $\bullet$ Fig. 1B).

The treatment with estrogens and 1,25D for 24h apparently did not exert any effect on the OPG secretion when we analyzed the joint data from the four patients. There was a decrease of between 14 and $58 \%$ in two patients and an increase of between 6 and $162 \%$ in the other two patients. Secondly, on analyzing 


\begin{tabular}{|c|c|c|c|c|}
\hline Molecule & Primers & Strand & Size (bp) & $\begin{array}{l}\text { Conditions } \\
\text { (Temp; no. cycles) }\end{array}$ \\
\hline OPG & $\begin{array}{l}\text { GAACCCCAGAGCGAAATACA } \\
\text { CGCTGTTTTCACAGAGGTCA }\end{array}$ & + & 441 & $54^{\circ} \mathrm{C} ; \times 25$ \\
\hline RANKL & $\begin{array}{l}\text { ATCCCATCTGGTTCCCATAA } \\
\text { CCCTGACCAATACTTGGTGC }\end{array}$ & $\begin{array}{l}+ \\
-\end{array}$ & 276 & $53.5^{\circ} \mathrm{C} ; \times 30$ \\
\hline ALP & $\begin{array}{l}\text { ACGTGGCTAAGAATGTCATC } \\
\text { CTGGTAGGCGATGTCCTTA }\end{array}$ & $\begin{array}{l}+ \\
-\end{array}$ & 475 & $52^{\circ} \mathrm{C} ; \times 30$ \\
\hline BGP & $\begin{array}{l}\text { CATGAGAGCCCTCACA } \\
\text { AGAGCGACACCCTAGAC }\end{array}$ & + & 310 & $52^{\circ} \mathrm{C} ; \times 30$ \\
\hline COL1 & $\begin{array}{l}\text { TGACGAGACCAAGAACTG } \\
\text { CCATCCAAACCACTGAAACC }\end{array}$ & $\begin{array}{l}+ \\
-\end{array}$ & 599 & $52^{\circ} \mathrm{C} ; \times 30$ \\
\hline$\beta$-actin & $\begin{array}{l}\text { TTGTAACCAACTGGGACGATATGT } \\
\text { GATCTTGATCTTCATGGTGCTAGG }\end{array}$ & $\begin{array}{l}+ \\
-\end{array}$ & 746 & $54^{\circ} \mathrm{C} ; \times 25$ \\
\hline
\end{tabular}

Table 1 Human oligonucleotide primers used for PCR. Primers are presented in a $5^{\prime}$ to $3^{\prime}$ orientation for coding $(+)$ and noncoding $(-)$ strands.

Note: Temperature and cycle numbers correspond to the linear part of the amplification curve
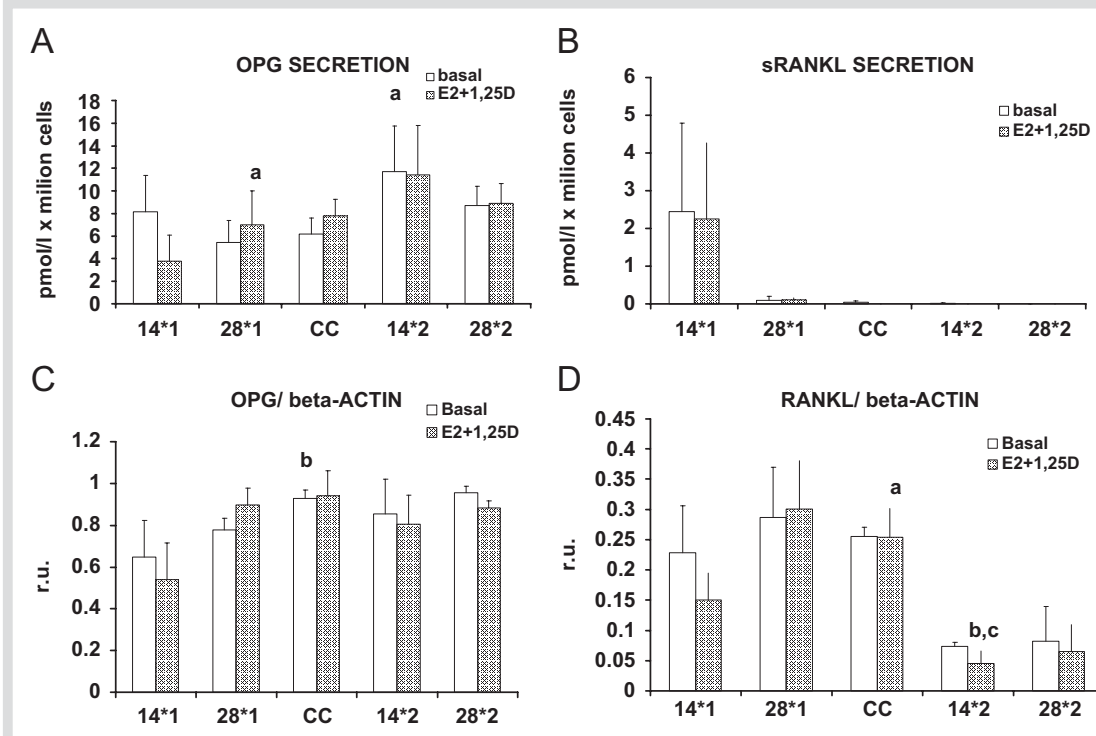

D

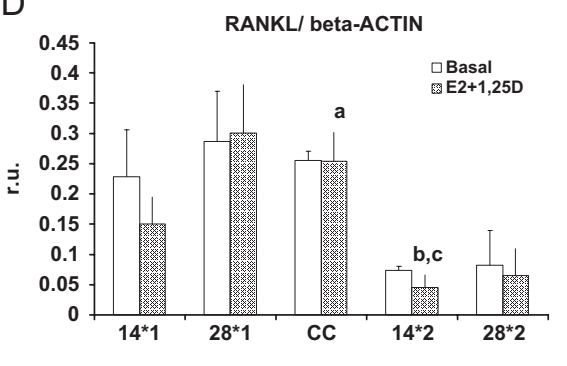

Fig. 1 Protein secretion and gene expression during in vitro maturation and differentiation of human primary osteoblasts (hOBs) in cultures under the influence of $10^{-6} \mathrm{M} 17$ - $\beta$-estradiol (E2) and $10^{-8} \mathrm{M}$ vitamin $\mathrm{D} 3(1,25 \mathrm{D})$ compared with control. Results are plotted in percentage of maximum secretion. (A) OPG protein secretion, (B) RANKL protein secretion. Gene expression was normalized with the expression of $\beta$-actin. (C) OPG gene expression, (D) RANKL gene expression. Mean values \pm SE of four experiments with two or three replicates; statistical significance: ${ }^{\mathrm{a}} \mathrm{p}<0.05$ vs. day 14 of first passage, ${ }^{b} p<0.05$ vs. day 28 of first passage, ${ }^{c} p<0.05$ vs. confluence. $14^{*} 1=$ day 14 of first passage; $28^{*} 1=$ day 28 of first passage; $\mathrm{CC}=$ cellular confluence; $14 * 2=$ day 14 of second passage; $28^{*} 2=$ day 28 of second passage.
SRANKL, while the levels were undetectable on the 28th day of the second passage in three out of four patients, after stimulating with $\mathrm{E} 2+1,25 \mathrm{D}$, the patients presented sRANKL secretion in the culture medium, thus indicating a positive net effect on this protein.

There was a negative correlation between the values of OPG and SRANKL secretion throughout cellular growth, with $r=-0.332$, $\mathrm{p}=0.036$.

\section{Osteoblast phenotype}

The mRNA levels were measured by semi-quantitative RT-PCR. The expression pattern of key genes such as COL1, ALP, and BGP was evident throughout the cellular culture, with higher concentrations for COL1 and ALP on the 28th day of the first passage and for BGP at the moment of cellular confluence in the first phase. Although no significant differences were found for any of the genes among the points studied, we were able to verify that the maximum increase in ALP values was 5 times the initial value, while it was 3.5 times for BGP expression. ( $\odot$ Fig. 2A-C). mRNA expression of OPG was nearly unchanged in two out of four patients, and the other two underwent a decrease in the range of $2.2-20 \%$. We observed no apparent change in RANKL expression.

\section{Osteoclastogenesis regulatory genes}

The OPG mRNA levels were measured by semi-quantitative RTPCR. These levels progressively increased throughout culture development until the 28th day of the second passage, when values were highest. The overall increase was $32 \%$, with significant differences between day 28 of the first passage and its cellular confluence in the same passage $(\mathrm{p}=0.031)$ ( $\odot$ Fig. 1C, Table 2 ).

Cellular stimulation with estrogens and 1,25dihydroxyvitamin D3 did not induce any apparent change in OPG expression during culture with regard to the results of cellular cultures in basal conditions.

We were able to observe a positive correlation between OPG mRNA and BGP mRNA values $(\mathrm{r}=0.716, \mathrm{p}=0.001)$.

RANKL expression kept within the same range of values in the first passage; however, it decreased significantly in the second passage, where values fell up to 3.5 times with respect to the maximum values obtained on day 28 of the first passage $(\mathrm{p}=0.024)$ ( Fig. 1D, Table 2). Once again, the E2 and 1,25D stimuli did not modify the RANKL expression throughout the study with regard to the values obtained in nontreated cells.

RANKL mRNA levels were correlated with those found for ALP $(\mathrm{r}=0.479, \mathrm{p}=0.012)$ and for COL1 $(\mathrm{r}=0.405, \mathrm{p}=0.029)$.

The modulation of osteoclastogenic activity is related to the RANKL/OPG ratio, and we observed that this ratio decreased during culture time until reaching the lowest values at the last point studied. This means that in the initial studies of cell growth, 


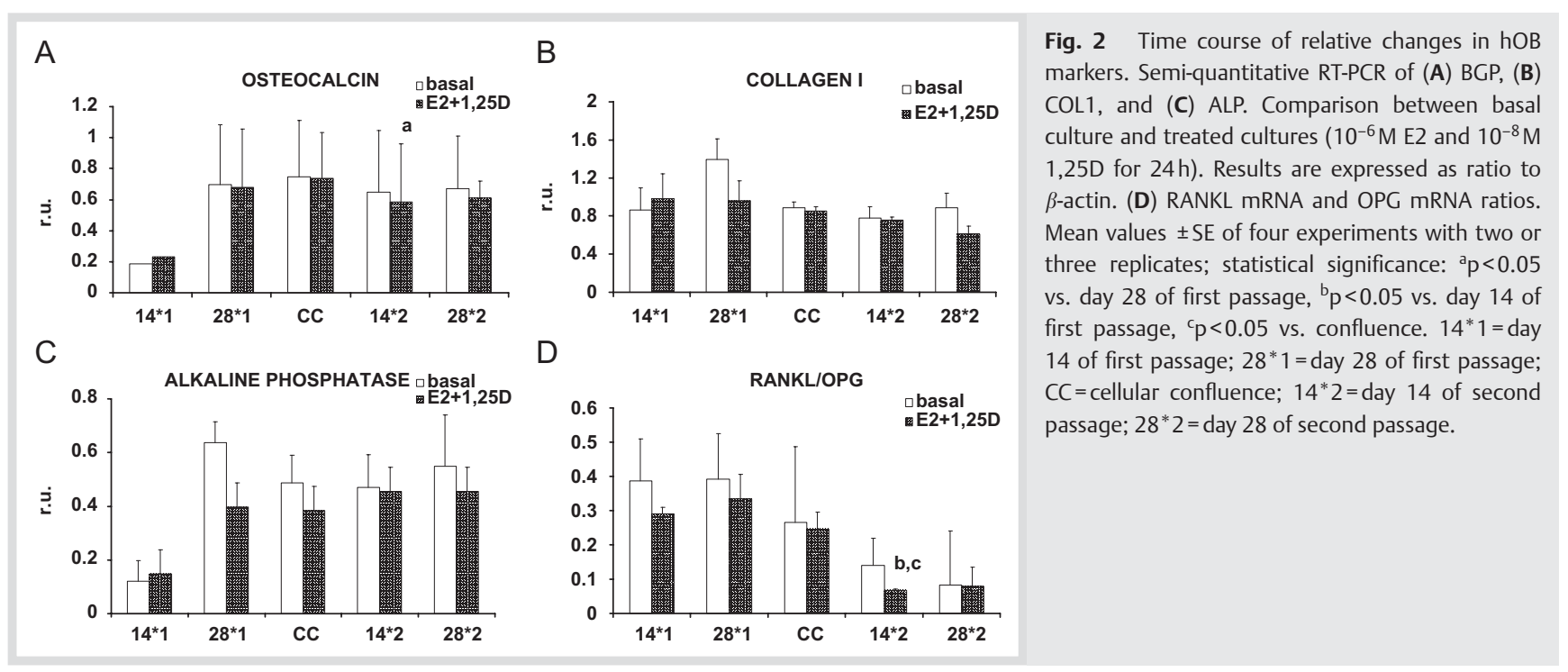

Table 2 Effect of $\beta$-estradiol and 1,25(OH) $2 \mathrm{D}_{3}(\mathrm{E} 2+\mathrm{VD})$ on OPG, RANKL expression (respect $\beta$-actin) and RANKL/OPG ratio. $14 * 1=14$ days of 1 st passage; $28^{*} 1=28$ days of 1 st passage; $C C=$ cellular confluence 1 st passage; $14^{*} 2=14$ days 2 nd passage; $28^{*} 2=28$ days 2 nd passage (r.u. relative unit).

\begin{tabular}{lllllll} 
& \multicolumn{2}{c}{ OPG (r.u.) } & \multicolumn{2}{c}{ RANKL (r.u.) } & \multicolumn{2}{c}{ RANKL/OPG } \\
& Basal & After E2+ VD & Basal & After E2 + VD & Basal & After E2+ VD \\
$\mathbf{1 4}^{*} \mathbf{1}$ & $0.65 \pm 0.2$ & $0.54 \pm 0.17$ & $0.23 \pm 0.08$ & $0.15 \pm 0.04$ & $0.39 \pm 0.1$ & $0.29 \pm 0.01$ \\
$\mathbf{2 8}^{*} \mathbf{1}$ & $0.78 \pm 0.05$ & $0.89 \pm 0.08$ & $0.29 \pm 0.08$ & $0.31 \pm 0.08$ & $0.39 \pm 0.1$ & $0.34 \pm 0.07$ \\
CC & $0.93 \pm 0.04^{*}$ & $0.94 \pm 0.12$ & $0.26 \pm 0.01^{*}$ & $0.25 \pm 0.05$ & $0.27 \pm 0.2$ & $0.25 \pm 0.05$ \\
$\mathbf{1 4}{ }^{*} \mathbf{2}$ & $0.85 \pm 0.1$ & $0.81 \pm 0.14$ & $0.07 \pm 0.01^{*}$ & $0.05 \pm 0.02$ & $0.14 \pm 0.1^{*}$ & $0.07 \pm 0.003$ \\
$\mathbf{2 8}^{*} \mathbf{2}$ & $0.96 \pm 0.03$ & $0.88 \pm 0.03$ & $0.08 \pm 0.05$ & $0.06 \pm 0.04$ & $0.08 \pm 0.1$ & $0.08 \pm 0.06$
\end{tabular}

Mean values \pm SE ; ${ }^{*} \mathrm{p}<0.05$

OBs modulate osteoclastogenic activity positively in the system (० Fig. 2D, Table 2).

\section{Discussion \\ $\nabla$}

In the present study we have shown that the patterns of different genes change throughout the time of hOBs cultures. The cultures were obtained from bone biopsies of adult patients who had undergone knee or hip arthrodesis. All of them similarly reached cellular confluence at 33-35d. During their growth, the percentage of cells obtained at each time of analysis was very similar. Confluence time was very similar to that obtained by other authors for primary osteoblastic cultures from hip bone biopsies [14-16], between 3 and 6 weeks, although it was more prolonged than that of other species such as rat primary OBs [17] or chicken embryo calvarial cells [18], which took 6-8d, or hOBs from other sources such as iliac crest biopsies [9], which reached cellular confluence after $21 \mathrm{~d}$ of culture.

There are three osteoblastic developmental phases [17]: an initial proliferative phase characterized by higher COL1 expression, followed by a matrix production and maturation phase in which ALP is increased, and, finally, a third phase of initiation of mineralization marked by higher levels of BGP mRNA. We detected COL1, ALP, and BGP levels throughout the culture growth up to cellular confluence. We were able to verify that the highest levels corresponded with day 28 of the first passage for the gene expression of COL1 and ALP. At the moment of cellular confluence for BGP mRNA in the second passage, the levels were main- tained, and we did not detect significant changes in the levels of mRNA of these genes. There are very few studies that have evaluated the expression of these genes in the first phases of cellular growth hOBs, and our results coincide with these studies $[11,14]$.

Expression of OPG and RANKL, key osteoblast regulatory genes, has hardly been studied throughout the development of the osteoblastic growth phases, although there are multiple authors who have studied OPG and RANKL expression at the moment of cellular confluence. Some have also studied OPG and RANKL protein secretion but found problems in detecting the latter $[12,19]$. Although the soluble form of sRANKL in the medium is well known [20,21], its detection has been difficult. We have detected soluble RANKL secretion in culture medium in the most precocious phases of cellular growth; in fact, only the determination carried out on the 14th day of the first culture passage showed sRANKL levels above the minimum threshold of sensibility of the test. Other authors have not found soluble levels of RANKL in the hOB culture medium, mainly because the SRANKL determinations were performed at later stages of cellular growth [22]. We did not evaluate the RANKL form anchored to a membrane in this study and, therefore, do not know whether this protein is modified throughout the differentiation phases and cellular maturation. Other studies will be necessary to determine this. In the present study we wanted to analyze protein secretion as well as OPG and RANKL expression during the growth of hOB cultures, first by evaluating the most incipient growth phases until cellular confluence was reached and then by relating the levels of these genes at the different time points 
with COL1, BGP, and ALP genes. Protein secretion as well as OPG expression were higher in studies of more immature cultures, coinciding with the BGP maximum peak (beginning of cellular maturation phase). These results have also been described in murine OBs in post-confluent growth [7] and in hOB cellular lines [23]. The behavior of sRANKL was different, with the highest secretion at the beginning of cellular culture and a sharp decrease from then onwards, reaching undetectable levels during the follow-up. This could explain the fact that some authors do not find a soluble form of the protein in the culture medium when they refer to the moment of cellular confluence $[19,22]$. RANKL gene expression was also greater at the beginning of cellular culture and coincided with ALP and COL1 expression, indicative of immature cells in the proliferative phase. These results are similar to the data shown by Gori et al. [23].

The RANKL/OPG mRNA ratio, indicative of positive modulation osteoclastogenic activity, showed higher values in the initial phases of the study, coinciding with more immature OBs, and decreased progressively in the latter phases of cellular maturation [23]. This makes us believe that the stimulation of osteoclastogenesis is a major role of immature osteoblasts. It would be necessary to perform, in the future, in vitro studies with both cellular types, osteoblasts and osteoclasts, and to observe this phenomenon throughout the different phases of cellular growth and development. Although the in vitro observations could not necessarily be extrapolated to in vivo physiologic mechanisms, we believe that the regulation of the RANKL/OPG ratio observed in vitro during cellular growth could contribute, in vivo, to the coordination of osteoblastic differentiation and osteoclastic activity.

E2 and 1,25D are hormones that influence bone turnover activity. Several in vitro studies have evaluated the effect each one has on the OPG/RANKL system, showing contradictory results $[19,24,25]$. In our case the results were not consistent, showing a stimulating effect on some cultures and inhibitory effects on others. We chose concentrations of $10^{-6} \mathrm{M}$ E2 and $10^{-8} \mathrm{M} 1,25 \mathrm{D}$ after performing previous studies of dose-response curves and observing that these concentrations showed higher activity. We used a combination of E2 and 1,25D because we were able to verify that the joint effect of both hormones induced more potent osteoblastic activity than each one did separately, coinciding with data from other authors $[26,27]$. This greater effect could be due to the upregulation of the estrogenic receptors by $1,25 \mathrm{D}$ when both substances are present, as other groups have proposed $[28,29]$. This led us to use a combination of both substances as a stimulus in the development of primary human cultures; however, we saw no relevant effect on the genes studied that could be related to the dose and time of incubation employed.

Overall, it appears that the differentiation over time is much stronger in regulating RANKL and OPG than E2 and 1,25D are. Thus, when assays to evaluate the effect of different pharmaceutical treatments on osteoblasts are designed, we have to bear in mind at what stage of proliferation and/or maturation the cell cultures are found [14].

In this work we did not measure the $\alpha$ or $\beta$ estrogen receptor (ER) concentrations in hOB cultures, which could limit our understanding of the results. Nevertheless, the existence of ER in OBs has been repeatedly observed, though at very low concentrations, which could justify the scarce response found [30-32]. In conclusion, in this study we have shown that human OB differentiation in vitro is associated with a high RANKL/OPG pro- duction ratio in early cellular growth stages $[23,33]$. We speculate that human OBs have a positive effect on modulating the osteoclastogenic activity at precocious stages of its cellular growth which is lost on reaching a higher degree of cellular maturation and that may contribute to the coordinated sequence of bone turnover cycles.

\section{Acknowledgments}

$\nabla$

This work was supported in part by a grant from Fondo de Investigación Sanitaria (FIS PI031164-2003) of Spain. The authors thank the personnel of the Traumatology Department and the Biochemistry Department at Hospital Virgen Macarena.

\section{References}

1 Roux S, Orcel P. Bone loss. Factors that regulate osteoclast differentiation: an update. Arthritis Res 2000; 2: 451-456

2 Manolagas SC, Jilka RL. Bone marrow, cytokines, and bone remodeling. Emerging insights into the pathophysiology of osteoporosis. N Engl J Med 1995; 332: 305-311

3 Khosla S. Minireview: the OPG/RANKL/RANK system. Endocrinology 2001; 142: 5050-5055

4 Kaji H, Kanatani M, Sugimoto T, Chihara K. Statins modulate the levels of osteoprotegerin/receptor activator of NFkappaB ligand mRNA in mouse bone-cell cultures. Horm Metab Res 2005; 37: 589-592

5 Shirai Y, Yoshimura Y, Yawaka Y, Hasegawa T, Kikuiri T, Takeyama S, Matsumoto A, Oguchi H. Effect of extracellular calcium concentrations on osteoclast differentiation in vitro. Biochem Biophys Res Commun 1999; 265: 484-488

6 Deyama Y, Takeyama S, Koshikawa M, Shirai Y, Yoshimura Y, Nishikata M, Suzuki K, Matsumoto A. Osteoblast maturation suppressed osteoclastogenesis in coculture with bone marrow cells. Biochem Biophys Res Commun 2000; 274: 249-254

7 Thomas GP, Baker SUK, Eisman JA, Gardiner EM. Changing RANKL/OPC mRNA expression in differentiating murine primary osteoblasts. J Endocrinol 2001; 170: 451-460

8 Stein GS, Lian JB. Molecular mechanisms mediating developmental and hormone-regulated expression of genes in osteoblasts: integrated relantionship of cell growth and differentiation. In: Noda M, ed. Cellular and molecular biology of bone. Tokyo: Academic Press 1993; 47-95

9 Siggelkow H, Rebenstorff K, Kurre W, Niedhart Ch, Engel I, Schilz H, Atkinson M, Hüfner M. Development of the osteoblast phenotype in primary human osteoblasts in culture: comparison with rat calvarial cells in osteoblast differentiation. I Cell Biochem 1999; 75: 22-35

10 Ishida H, Bellows CG, Aubin JE, Heersche JN. Characterization of the 1,25- $(\mathrm{OH}) 2 \mathrm{D} 3$-induced inhibition of bone nodule formation in longterm cultures of fetal rat calvaria cells. Endocrinology 1993; 132: 61-66

11 Siggelkow H, Schulz H, Kaesler S, Benzler K, Atkinson MJ, Hüfner M. 1,25 dihydroxyvitamin-D3 attenuates the confluencedependent differences in the osteoblast characteristic proteins alkaline phosphatase, procollagen I peptide, and osteocalcin. Calcif Tissue Int 1999; 64: 414-421

12 Bord S, Ireland DC, Beavan SR, Compston JE. The effects of estrogen on osteoprotegerin, RANKL, and estrogen receptor expression in human osteoblasts. Bone 2003; 32: 136-141

13 Pascher E, Perniok A, Becker A, Feldkamp J. Effect of 1alpha,25(OH)2vitamin D3 on TNF alpha-mediated apoptosis of human primary osteoblast-like cells in vitro. Horm Metab Res 1999; 31: 653-656

14 Spreafico A, Frediani B, Capperucci C, Chellini F, Paffetti A, D'Ambrosio C Bernardini G, Mini R, Collodel G, Scaloni A, Marcolongo R, Santucci A. A proteomic study on human osteoblastic cells proliferation and differentiation. Proteomics 2006; 6: 3520-3532

15 Im GI, Qureshi SA, Kenney J, Rubash HE, Shanbhag AS. Osteoblast proliferation and maturation by bisphosphonates. Biomaterials 2004; 25 : 4105-4115

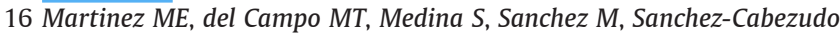
MJ, Esbrit P, Martinez P, Moreno I, Rodrigo A, Garces MV, Munuera L. Influence of skeletal site of origin and donor age on osteoblastic cell growth and differentiation. Calcif Tissue Int 1999; 64: 280-286

17 Owen TA, Aronow MS, Barone LM, Bettencourt B, Stein GS, Lian JB. Pleiotropic effects of vitamin D on osteoblast gene expression are 
related to the proliferative and differentiated state of the bone cell phenotype: dependency upon basal levels of gene expression, duration of exposure, and bone matrix competency in normal rat osteoblast cultures. Endocrinology 1991; 128: 1496-1504

18 Gerstenfeld LC, Chipman SD, Glowacki J, Lian JB. Expression of differentiated function by mineralizing cultures of chicken osteoblasts. Dev Biol 1987; 122: 49-60

19 Cheung J, Mak YT, Papaioannou S, Evans BA, Fogelman I, Hampson G. Interleukin-6 (IL-6), IL-1, receptor activator of nuclear factor kappaB ligand (RANKL) and osteoprotegerin production by human osteoblastic cells: comparison of the effects of 17-beta oestradiol and raloxifene. J Endocrinol 2003; 177: 423-433

20 Burgess TL, Qian Y, Kaufman S, Ring BD, Van G, Capparelli C, Kelley M, Hsu H, Boyle WJ, Dunstan CR, Hu S, Lacey DL. The ligand for osteoprotegerin (OPGL) directly activates mature osteoclasts. J Cell Biol 1999; 145: 527-538

21 Skoumal M, Kolarz G, Haberhauer G, Woloszczuk W, Hawa G, Klingler A. Osteoprotegerin and the receptor activator of NF-kappa $B$ ligand in the serum and synovial fluid. A comparison of patients with longstandin rheumatoid arthritis and osteoarthritis. Rheumatol Int 2005; 26: 63-69

22 Viereck V, Grundker C, Blaschke S, Siggelkow H, Emons G, Hofbauer LC. Phytoestrogen genistein stimulates the production of osteoprotegerin by human trabecular osteoblasts. J Cell Biochem 2002; 84: 725-735

23 Gori F, Hofbauer LC, Dunstan CR, Spelsberg TC, Khosla S, Riggs BL. The expression of osteoprotegerin and RANK ligand and the support of osteoclast formation by stromal-osteoblast lineage cells is developmentally regulated. Endocrinology 2000; 141: 4768-4776

24 Chen $Q$ Kaji $H$, Kanatani $M$, Sugimoto T, Chihara K. Testosterone increases osteoprotegerin mRNA expression in mouse osteoblast cells. Horm Metab Res 2004; 36: 674-678

25 Baldock PA, Thomas GP, Hodge JM, Baker SU, Dressel U, O'Loughlin PD, Nicholson GC, Briffa KH, Eisman JA, Gardiner EM. Vitamin d action and regulation of bone remodeling: suppression of osteoclastogenesis by the mature osteoblast. J Bone Miner Res 2006; 21: 1618-1626
26 Picotto G. Rapid effects of calciotropic hormones on female rat enterocytes: combined actions of 1,25(OH)2-vitamin D3, PTH and 17betaestradiol on intracellular $\mathrm{Ca}^{2+}$ regulation. Horm Metab Res 2001; 33: 733-738

27 Chen FP, Lee $N$, Wang $K C$, Soong $Y K$, Huang KE. Effect of estrogen and 1alpha,25(OH)2- vitamin D3 on the activity and growth of human primary osteoblast-like cells in vitro. Fertil Steril 2002; 77: 1038-1043

28 Bellido T, Girasole G, Passeri G, Yu XP, Mocharla H, Jilka RL, Notides A, Manolagas SC. Demonstration of estrogen and vitamin D receptors in bone marrow-derived stromal cells: up-regulation of the estrogen receptor by 1,25-dihydroxyvitamin-D3. Endocrinology 1993; 133: 553-562

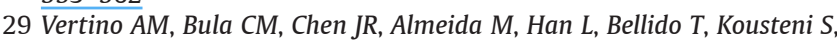
Norman AW, Manolagas SC. Nongenotropic, anti-apoptotic signaling of 1alpha,25(OH)2-vitamin D3 and analogs through the ligand binding domain of the vitamin D receptor in osteoblasts and osteocytes. Mediation by Src, phosphatidylinositol 3-, and JNK kinases. J Biol Chem 2005; 280: 14130-14137

30 Arts J, Kuiper GG, Janssen JM, Gustafsson JA, Löwik CW, Pols HA, Leeuwen JP van. Differential expression of estrogen receptors alpha and beta mRNA during differentiation of human osteoblast SV-HFO cells. Endocrinology 1997; 138: 5067-5070

31 Bord S, Horner A, Beavan S, Compston J. Estrogen receptors alpha and beta are differentially expressed in developing human bone. J Clin Endocrinol Metab 2001; 86: 2309-2314

32 Vanderschueren D, Vandenput L, Boonen S, Lindberg $M K$, Bouillon $R$, Ohlsson C. Androgens and bone. Endocr Rev 2004; 25: 389-425

33 Parfitt AM. Osteonal and hemi-osteonal remodeling: the spatial and temporal framework for signal traffic in adult human bone. J Cell Biochem 1994; 55: 273-286 